\title{
Reliability Contingency Analysis by Static Synchronous Series Compensator in Optimal Power Flow
}

\author{
Sanjoy Kumar Saha
}

\begin{abstract}
One of the best innovated Flexible A.C Transmission system (FACTS) devices is the static synchronous series compensator (SSSC).This devices is effective to alter power system parameter in order to increase power transfer capacity, stabilize system. This paper show how may reduce the generation coast. These FACTS devices enable a continues and wear resistance control of reactive power flows as well as voltage control. The incorporate of the control capabilities provided by FACTS devices into an optimal power flow open up an unused potential for reducing power losses as well as coast for power transmission of power system operator.
\end{abstract}

Index Terms- FACTS, Optimal Power Flow (OPF), Static Synchronous Series Compensator (SSSC)

\section{INTRODUCTION}

Recent developments in semiconductor technology have made the Flexible A.C Transmission system (FACTS) devices [5], [6] of special interest to power system operation and transmission providers. Their operation are realized using advanced power electronics components which make them response quickly to the control inputs, and their instant response grants a high ability for power system dynamic stability enhancement in addition to control of steady-state power flow[5]-[15].

Utilizing the powerful FACTS devices may help interconnected system have increased power transfer capability for the existing power transmission lines due to the constantly increasing loads the power networks are required to serve. The optimization tasks in a competitive environment comprises in particular coast minimizing including different aspects of the procurement and transmission of electrical energy or the reduction of power losses. Alternatively the optimization aims at maximization of the available transmission capacity.

A SSSC offers a fast series compensation offer flexible power system control [6], therefore, it can be utilized to control line active and reactive power, achieve more power transfer capability, and so significantly improved power system stability. As the SSSC is capable of controlling power system parameters.

These papers reviews the structure of SSSC and derive the exact Pi-model of the SSSC embedded transmission lines from which the injection model using two port network is presented. These of SSSC also review some of the possible uses and advantages of SSSC in power system operations. The generalized formulation of an optimal power flow including exact model of SSSC. Because the unified power flow controller is the most versatile FACTS-device which allow the independent influence of active and reactive power flows as well as a control of the voltage profile at the same time.

Unified power flow controller (UPFC) is a combination of STATCOM (Static Synchronous Compensator) and SSSC which are coupled via a common dc link to allow bidirectional flow of power between the series output terminals of the SSSC and the shunt output terminals of the STATCOM. These are controlled to provide concurrent real and reactive series line compensation without and external energy source.

The UPFC, by means of angularly unconstrained series voltage injection, is able to control, concurrently and selectively, the transmission line voltage, impedance and angle or alternatively the real and reactive power flow in the line. The UPFC may also provide independently controllable shunt reactive compensation. A UPFC as show in fig (1) .This is a complete controller for controlling active and reactive power control through the line, as well as line volt control.

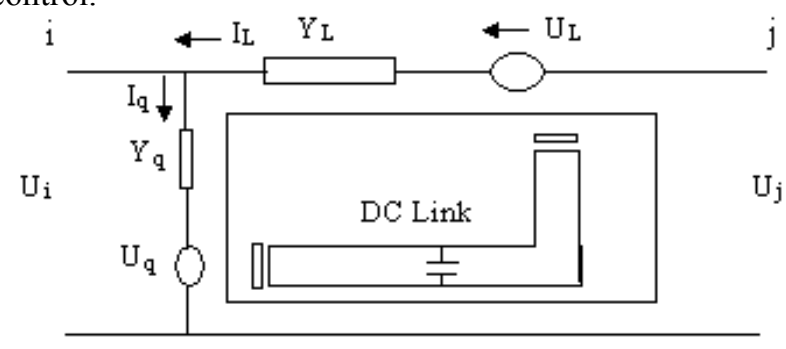

Fig.(1) UPFC voltage source model

\section{Structure, Moduling OF SSSC}

1) Structure of $\mathrm{SSSC}$

Fig (2) represented a SSSC inserted in the line connecting i \& j.

A SSSC is basically consisting of converter with semiconductor devices having turn off capability, a coupling transformer and a capacitor [16]-[18].A converter is connect to a power system through the coupling transformer. The DC capacitor provides a DC voltage support for the converter operator and functions as an energy storage element. The SSSC injection a voltage in series with transmission line through the series transformer. 


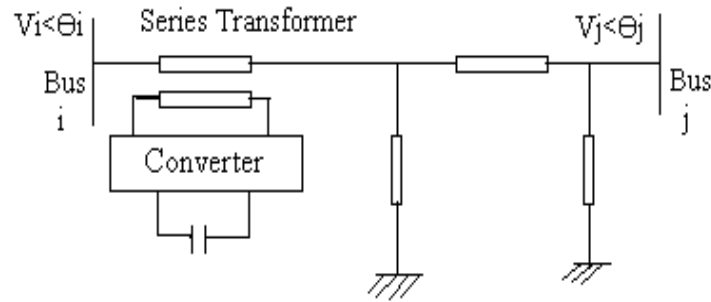

Fig(2).Schematic diagram of SSSC inserted transmission line.

Two part model of SSSC-Embedded line Fig(3) represented as SSSC circuit model.

The SSSC is located between $\mathrm{i}$ and $\mathrm{m}$. The equivalent circuit includes both the two port steady state model of the SSSC and the two port Pi model of the transmission line connected in cascade .The converter is represented by a voltage source in series impedance, where $Z_{\mathrm{se}}\left(\mathrm{Y}_{\mathrm{se}}\right)$ is the impedance (admittance) associate with the series converter, Where $\mathrm{Y}_{\mathrm{se}}=\mathrm{G}_{\mathrm{se}}+\mathrm{j} \mathrm{B}_{\mathrm{se}}$. The complex voltage $\mathrm{V}_{\mathrm{se}}$ is the controllable voltage inserted from the converter, and $\mathrm{V}_{\mathrm{i}}$ and $\mathrm{V}_{\mathrm{j}}$ are the complex voltage at voltage at node $\mathrm{I}$ and $\mathrm{j}$, respectively .

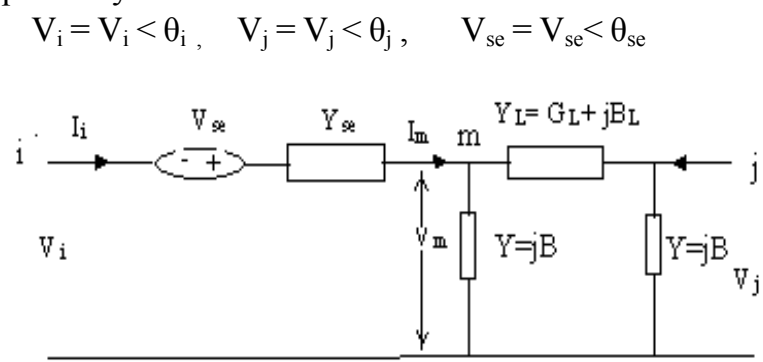

Fig(3) Representing the SSSC Model

From the circuit of fig(3). We can write the following relation

$$
\left[\begin{array}{c}
V_{i} \\
I_{i}
\end{array}\right]=\left[\begin{array}{cc}
1 & Z_{s e} \\
0 & 1
\end{array}\right]\left[\begin{array}{l}
V_{m} \\
I_{m}
\end{array}\right]+\left[\begin{array}{l}
1 \\
0
\end{array}\right] V_{s e}
$$

Let $V_{L}=G_{L}+j B_{L}$ and $Y=j B$ are the admittance of the two port network of $\mathrm{Pi}$-equivalent circuit of a transmission line in fig (3). The transmission line is described by the following equation.

$$
\left[\begin{array}{c}
I_{m} \\
I_{j}
\end{array}\right]=\left[\begin{array}{cc}
\left(Y_{L}+Y\right) & Y_{L} \\
Y_{L} & \left(Y_{L}+Y\right)
\end{array}\right]\left[\begin{array}{c}
V_{m} \\
I_{j}
\end{array}\right]
$$

From three we can express $I_{m}$ and $I_{j}$ in term of $V_{m}$ and $V_{j}$ as follows

$$
\left[\begin{array}{c}
I_{m} \\
I_{j}
\end{array}\right]=\left[\begin{array}{cc}
\left(Y_{L}+Y\right) & Y_{L} \\
Y_{L} & \left(Y_{L}+Y\right)
\end{array}\right]\left[\begin{array}{c}
V_{m} \\
I_{j}
\end{array}\right]
$$

Let $\mathrm{Y}_{\mathrm{T}}=\mathrm{Yse}+\mathrm{Y}_{\mathrm{L}}+\mathrm{Y}$, then for the circuit of fig (3), we can express $I_{i}$ and $I_{j}$ in terms of $V_{i}$ and $V_{j}$, and $V_{\text {se }}$ in matrix form follows:

$$
\left[\begin{array}{c}
I_{i} \\
I_{j}
\end{array}\right]=\left[\begin{array}{ll}
Y_{i i} & Y_{i j} \\
Y_{j i} & Y_{j j}
\end{array}\right]\left[\begin{array}{l}
V_{i} \\
V_{j}
\end{array}\right]+\left[\begin{array}{l}
b_{i} \\
b_{j}
\end{array}\right] V_{s e}
$$

Where $Y_{i j}=Y_{j i}=-Y_{\text {se }}\left(Y_{L} / Y_{T}\right)$

$$
\begin{aligned}
& Y_{\mathrm{ii}}=\mathrm{G}_{\mathrm{ii}}+\mathrm{jB}_{\mathrm{ii}}=\left(\mathrm{Y}_{\mathrm{se}} \mathrm{Y}_{\mathrm{T}}-\mathrm{Y}_{\mathrm{se}}{ }^{2}\right) / \mathrm{Y}_{\mathrm{T}} \\
= & \mathrm{Y}_{\mathrm{se}} \mathrm{Y}_{\mathrm{L}} / \mathrm{Y}_{\mathrm{T}}+\left(\mathrm{Y}_{\mathrm{se}} \mathrm{Y}\right) / \mathrm{Y}_{\mathrm{T}}=-\mathrm{Y}_{\mathrm{ij}}+\mathrm{Y}_{\mathrm{io}}
\end{aligned}
$$

$$
\begin{gathered}
Y_{j j}=Y_{s e} Y_{L} / Y_{T}+Y\left(Y_{s e}+2 Y_{L}+Y\right) / Y_{T}=-Y_{i j}+Y_{j o} \\
b_{i}=G_{b i}+j B_{b i}=Y_{s e}\left(1-Y_{s e} / Y_{T}\right)=Y_{s e}\left(Y_{L}+Y\right) / Y_{T} \\
b_{j}=G_{b j}+j B_{b j}=-Y_{s e} Y_{L} / Y_{T}=Y_{j i}
\end{gathered}
$$

Note that we decomposed each of $Y_{\mathrm{ii}}$ and $\mathrm{Y}_{\mathrm{jj}}$ into two parts, i.e,

$$
\begin{gathered}
Y i i=-Y i j+Y \text { io and } Y j j=-Y i j+Y j o, \quad \text { where; } \\
Y_{i i}=G_{i j}+j B_{i j}=-Y_{\text {se }} Y_{L} / Y_{T} \\
Y_{j o}=G_{i o}+j B_{j o}=-Y_{s e} Y_{L} / Y_{T} \\
Y_{j o}=G_{j o}+j B_{j o}=Y\left(Y_{s e}+2 Y_{L}+Y\right) / Y_{T}
\end{gathered}
$$

Equation (5) and (6)-(10) can be represented by the circuit in fig (4). The fig. represents modeling of the SSSC embedded line using current source and modified admittances.

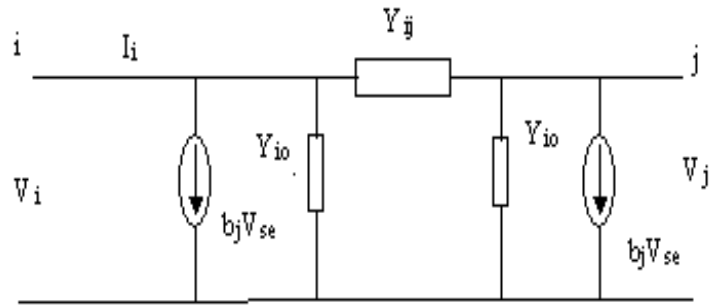

Fig(4) SSSC model using current source.

\section{LOAD FLOW EQUATION OF PI-MODEL.}

The complex power injection Pi-model of the SSSC embedded line, which is derived from fig(4), is show in fig (5). The complex power $\mathrm{S}_{\mathrm{i}}^{\text {se }}$ at bus $\mathrm{i}$ and $\mathrm{S}_{\mathrm{j}}^{\mathrm{se}}$ at bus $\mathrm{j}$ represents complex power associated with the current source show in fig (4). To derive the complex power injections and flows of the Pi-model of the SSSC embedded line, the withdrawals due to SSSC at bus $i$ and $j$ are calculated as

$$
\begin{aligned}
& \mathrm{S}_{\mathrm{i}}^{\mathrm{se}}=\mathrm{V}_{\mathrm{i}}\left(\mathrm{b}_{\mathrm{i}} \mathrm{V}_{\mathrm{se}}\right)^{*} \text { and } \mathrm{S}_{\mathrm{j}}^{\mathrm{se}}=\mathrm{V}_{\mathrm{j}}\left(\mathrm{b}_{\mathrm{j}} \mathrm{V}_{\mathrm{se}}\right)^{*} \\
& \mathrm{~S}_{\mathrm{i}}^{\mathrm{se}}=\mathrm{P}_{\mathrm{i}}^{\mathrm{se}}+\mathrm{jQ}_{\mathrm{i}}{ }^{\mathrm{se}}=\mathrm{V}_{\mathrm{i}}<\Theta_{\mathrm{i}}\left(\left(\mathrm{G}_{\mathrm{bi}}+\mathrm{jB}_{\mathrm{bi}}\right) \mathrm{V}_{\mathrm{se}}<\delta\right)^{*} \\
& \mathrm{~S}_{\mathrm{j}}{ }^{\mathrm{se}}=\mathrm{P}_{\mathrm{j}}{ }^{\mathrm{se}}+\mathrm{j} \mathrm{Q}_{\mathrm{j}}{ }^{\mathrm{se}}=\mathrm{V}_{\mathrm{j}}<\Theta_{\mathrm{j}}\left(\left(\mathrm{G}_{\mathrm{bj}}+\mathrm{jB}_{\mathrm{bj}}\right) \mathrm{V}_{\mathrm{se}}<\delta\right)^{*}
\end{aligned}
$$

From equation (14) and (15), we can write

$$
\begin{array}{r}
\mathrm{P}_{\mathrm{i}}^{\mathrm{se}}=\mathrm{V}_{\mathrm{i}} \mathrm{V}_{\mathrm{se}}\left[-\mathrm{B}_{\mathrm{bj}} \cos \left(\Theta_{\mathrm{i}}-\delta\right)+\mathrm{G}_{\mathrm{bi}} \sin \left(\Theta_{\mathrm{i}}-\delta\right)\right] \\
\mathrm{Q}_{\mathrm{i}}^{\mathrm{se}=}=\mathrm{V}_{\mathrm{i}} \mathrm{V}_{\mathrm{se}}\left[\mathrm{G}_{\mathrm{bj}} \cos \left(\Theta_{\mathrm{i}}-\delta\right)+\mathrm{B}_{\mathrm{bi}} \sin \left(\Theta_{\mathrm{i}}-\delta\right)\right] \\
\mathrm{P}_{\mathrm{j}}^{\mathrm{se}}=\mathrm{V}_{\mathrm{j}} \mathrm{V}_{\mathrm{se}}\left[\mathrm{G}_{\mathrm{bj}} \cos \left(\Theta_{\mathrm{j}}-\delta\right)+\mathrm{B}_{\mathrm{bj}} \sin \left(\Theta_{\mathrm{j}}-\delta\right)\right. \\
\mathrm{Q}_{\mathrm{j}}^{\mathrm{se}}=\mathrm{V}_{\mathrm{j}} \mathrm{V}_{\mathrm{se}}\left[-\mathrm{B}_{\mathrm{bj}} \cos \left(\Theta_{\mathrm{j}}-\delta\right)+\mathrm{G}_{\mathrm{bj}} \sin \left(\Theta_{\mathrm{j}}-\delta\right)\right]
\end{array}
$$

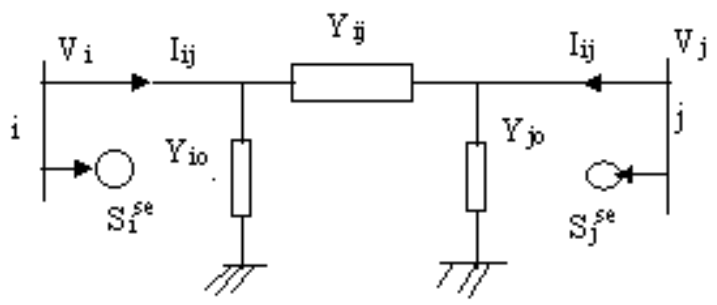

Fig(5) The injection Pi-model of the SSSC Embedded line.

The current $\mathrm{I}_{\mathrm{ij}}$ and $\mathrm{I}_{\mathrm{ji}}$ and complex power $\mathrm{S}_{\mathrm{ij}}\left(\mathrm{S}_{\mathrm{ij}}=\mathrm{V}_{\mathrm{i}} \mathrm{I}_{\mathrm{ij}}{ }^{*}\right)$ and $\mathrm{S}_{\mathrm{ji}}\left(\mathrm{S}_{\mathrm{ji}}=\mathrm{V}_{\mathrm{j}} \mathrm{I}_{\mathrm{ji}}{ }^{*}\right)$ flowing out of buses in the line $\mathrm{i}-\mathrm{j}$ can be expressed as

$\mathrm{I}_{\mathrm{ij}}=\left(\mathrm{V}_{\mathrm{i}}-\mathrm{V}_{\mathrm{j}}\right) \mathrm{V}_{\mathrm{ij}}+\mathrm{V}_{\mathrm{i}} \mathrm{Y}_{\mathrm{io}}==\left(\mathrm{V}_{\mathrm{i}}-\mathrm{V}_{\mathrm{j}}\right)\left(\mathrm{G}_{\mathrm{ij}}+\mathrm{j} \mathrm{Bij}\right)+\mathrm{V}_{\mathrm{i}}\left(\mathrm{G}_{\mathrm{io}}+\mathrm{jB}_{\mathrm{io}}\right)$

$\mathrm{I}_{\mathrm{ji}}=\left(\mathrm{V}_{\mathrm{j}}-\mathrm{V}_{\mathrm{i}}\right) \mathrm{V}_{\mathrm{ji}}+\mathrm{V}_{\mathrm{j}} \mathrm{Y}_{\mathrm{jo}}==\left(\mathrm{V}_{\mathrm{i}}-\mathrm{V}_{\mathrm{j}}\right)\left(\mathrm{G}_{\mathrm{ij}}+\mathrm{j} \mathrm{B}_{\mathrm{ij}}\right)+\mathrm{V}_{\mathrm{j}}\left(\mathrm{G}_{\mathrm{jo}}+\mathrm{j} \mathrm{B}_{\mathrm{jo}}\right)$

$S_{i j}=G_{i j} \quad\left[V_{i}^{2}-V_{i} V_{j} \quad \cos \left(\Theta_{i}-\Theta_{j}\right)\right]-B_{i j}\left[V_{i} V_{j} \sin \left(\Theta_{i}-\Theta_{j}\right)\right]+G_{i o} V_{i}^{2}$ 
$-\mathrm{j}\left\{\mathrm{B}_{\mathrm{ij}}\left[\mathrm{V}_{\mathrm{i}}^{2}-\mathrm{V}_{\mathrm{i}} \mathrm{V}_{\mathrm{j}} \cos \left(\Theta_{\mathrm{i}}-\Theta_{\mathrm{j}}\right)\right]+\mathrm{G}_{\mathrm{ij}}\left[\mathrm{V}_{\mathrm{i}} \mathrm{V}_{\mathrm{j}} \sin \left(\Theta_{\mathrm{i}}-\Theta_{\mathrm{j}}\right)\right]+\mathrm{B}_{\mathrm{io}} \mathrm{V}_{\mathrm{i}}^{2}\right\}(20)$

$S_{j i}=G_{i j}\left[V_{j}^{2}-V_{j} V_{i} \cos \left(\Theta_{j}-\Theta_{i}\right)\right]-B_{i j}\left[V_{j} V_{i} \sin \left(\Theta_{j}-\Theta_{i}\right)\right]+G_{j o} V_{j}^{2}$ $-\mathrm{j}\left\{B_{\mathrm{ij}}\left[\mathrm{V}_{\mathrm{j}}^{2}-\mathrm{V}_{\mathrm{j}} \mathrm{V}_{\mathrm{i}} \cos \left(\Theta_{\mathrm{j}}-\Theta_{\mathrm{i}}\right)\right]+\mathrm{G}_{\mathrm{ij}}\left[\mathrm{V}_{\mathrm{j}} \mathrm{V}_{\mathrm{i}} \quad \sin \left(\Theta_{\mathrm{j}}-\Theta_{\mathrm{i}}\right)\right]+\mathrm{B}_{\mathrm{jo}} \mathrm{V}_{\mathrm{j}}^{2}\right\}$ $(21)$

Let $R_{i}$ is set of buses connected to bus $i, P_{i} G / Q_{i} G$ is the active/reactive power generated at bus $\mathrm{i}, \mathrm{P}_{\mathrm{i}}^{\mathrm{D}} / \mathrm{Q}_{\mathrm{i}}^{\mathrm{D}}$ is the active/reactive power demanded at bus $i$, and $\mathrm{P}_{i}{ }^{\circ} / \mathrm{Q}_{i}{ }^{\circ}$ is the net active/reactive power flowing out bus $i$, then for $i, j$ $=1,2 \ldots \ldots . . \mathrm{NB}$, Where NB is the number of buses, the load flow balance equations can be expressed as follows .

$$
\begin{aligned}
& \mathrm{P}_{\mathrm{i}}^{\mathrm{G}}-\mathrm{P}_{\mathrm{i}}{ }^{\mathrm{D}}=\mathrm{P}_{\mathrm{i}}^{\mathrm{se}}+\mathrm{P}_{\mathrm{i}}{ }^{\mathrm{o}} \\
& \mathrm{Q}_{\mathrm{i}}{ }^{\mathrm{G}}-\mathrm{Q}_{\mathrm{i}}^{\mathrm{D}}=\mathrm{Q}_{\mathrm{i}}^{\mathrm{se}}+\mathrm{Q}_{\mathrm{i}}{ }^{\mathrm{O}}
\end{aligned}
$$

$$
Q_{i}^{o}=-V_{i}^{2} b_{i i} \sum_{j \in R_{i}} V_{i} V_{j}\left[b_{i j} \cos \left(\theta_{i}-\theta_{j}\right)+g_{i j} \sin \left(\theta_{i}-\theta_{j}\right)\right]
$$

The current $I_{m}$ in the fig.(3) can be found using any of the following equation .

$$
I_{m}=Y_{s e}\left[\left(Y+Y_{L}\right)\left(V_{i}+V_{s e}\right)-Y_{L} V_{j}\right] /\left(Y+Y_{L}+Y_{s e}\right)
$$

\section{Optimal Power Flow}

Optimal power flow expresses as follows:

$$
f(x)=f\left(P^{G}, Q^{G}, V, \theta, V_{s e}, \delta\right)
$$

Subject to

Equality constraints:

$P_{i}^{G}-P_{i}^{D}=P_{i}^{s e}+P_{i}^{o}$

$Q_{i}^{G}-Q_{i}^{D}=Q_{i}^{s e}+Q_{i}^{o}$

$P_{j}^{G}-P_{j}^{D}=P_{j}^{s e}-P_{j}^{o}$

$Q_{j}^{G}-Q_{j}^{D}=Q_{j}^{s e}-Q_{j}^{o}$

Inequality constraints:

$P_{i j}^{\min } \leq P_{i j} \leq P_{i j}^{\max }, p_{j i}^{\min } \leq P_{j i} \leq P_{j i}^{\max }$

$P_{i}^{G_{\text {min }}} \leq P_{i}^{G} \leq P_{i}^{G_{\text {max }}}$

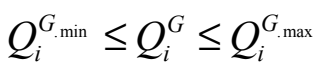

$V_{i}^{\min } \leq V_{i} \leq V_{i}^{\max }$

$V_{s e}^{\min } \leq V_{s e} \leq V_{s e}^{\max }$

\begin{tabular}{|c|c|c|c|c|c|c|c|c|c|c|c|}
\hline & 웅 & 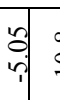 & $\begin{array}{ll}0 & \infty \\
= & \infty \\
4\end{array}$ & $=$ & :- & & 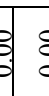 & 8 & & & 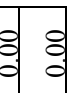 \\
\hline & ? & & & $\approx$ & 웡 & & & $\stackrel{\circ}{\circ}$ & & & 8 \\
\hline & $\stackrel{\infty}{+}$ & & 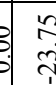 & & $\stackrel{\text { cి }}{\rightarrow}$ & & & $\stackrel{8}{0}$ & & & 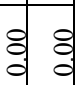 \\
\hline & : & & 8 & & a & : & & $\stackrel{0}{0}$ & & & \\
\hline & : & & 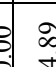 & & : & $\sigma$ & & $\stackrel{1}{0}$ & & & \\
\hline & & 8 & & & 의 & of & & 8 & & & \\
\hline & 9. & 0 & 0 & & 9 & 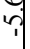 & & 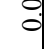 & $\therefore$ & & \\
\hline & : & $:$ & $b_{7}^{\infty}$ & & : & ? & 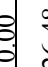 & $\stackrel{\infty}{=}$ & : & & \\
\hline & \& & 8 & 58 & $\&$ & \& & 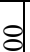 & 8 & to. & $\vec{\sim}$ & & \\
\hline & & 0 & & o & 0 & & & $\approx$ & 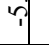 & & \\
\hline & : & $\stackrel{8}{\circ}$ & $\stackrel{0}{0}$ & $\stackrel{8}{\circ}$ & $\begin{array}{l}0 \\
4\end{array}$ & $\varepsilon$ & a & त्र & $\stackrel{\overbrace{}}{\Omega}$ & & \\
\hline & 의 & 8 & & & न & $\varepsilon$ & 8 & 8 & 8 & & \\
\hline & & & & 0 & i) & & & & & & \\
\hline & 웅 & $\stackrel{8}{\circ}$ & $\stackrel{8}{0}$ & $\stackrel{0}{\circ}$ & 연 & & 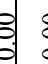 & $\stackrel{8}{0}$ & : & & \\
\hline & 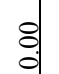 & 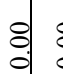 & $\approx$ & & & & & & & & \\
\hline
\end{tabular}

$\delta^{\min } \leq \delta \leq \delta^{\max }$

\section{TEST RESULT}

Input IEEE-14 data has been taken from M A PAI[18]

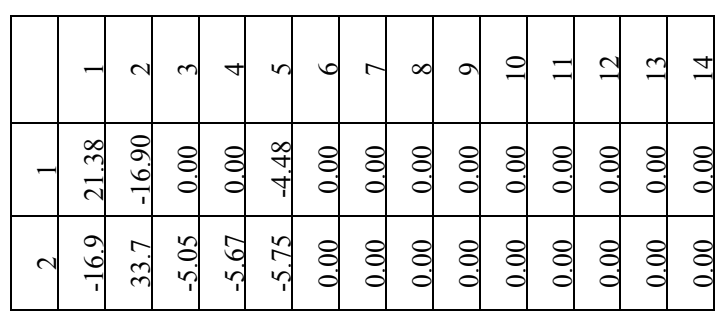

B-bus Matrix

\section{CONCLUSION}

The presented approach combines suitable linear models for facts-devices with a powerful optimization model including security constraints which are of major importance when optimizing existing power systems within the new frame work.

The SSSC offers a fast series compensation and grant flexible power system control by adjusting the basic power system parameters on which system performance depends. Therefore, it can be utilized to control line active and reactive power flows achieve more power transfer capability for the existing power network.

\section{REFERENCES}

[1] B. A. Renz, A. Keri, A. S. Mehraban, C. Schauder, E. Stacey, L. Ko-valsky, L. Gyugyi, and A. Edris, "AEP unified power flow controller performance," IEEE Trans. Power Syst., vol. 14, pp. 1374-1381, Oct.1999.

[2] D. J. Christofersen, H. Elahi, and M. G. Bennett, "A survey of the reli-ability of HVDC systems throughout the world during 1995-1996," in Proc. Cigré Session, Paper 14-102, 1998.

[3] E. Handschin and C. Lehmköster, "Optimal power flow for deregulated systems with FACTS-devices," in Proc. 13th Power Syst. Computat.Conf., Trondheim, Norway, pp. 1270-1276

[4] L. Gvunvi. C. D. Shaudsr. and K. K. Sen. "Slalic svnchronous series ,_comprnsator: A salid-statc approach to the series cornpcnsalion of transniissiun lincs." IEEE Trans. Power Urlrvery. vol. I2.406-413. Jan. 1997.

[5] N. G. Hingoran and I.. Gyuqyi. Understanding FAC7S. IEEE Press. 2000

[6] Y. H. Song and A. Johns. Flexrble AC Troransm,ssion Systems (FACTS),IEE, 1999 
[7] S.Y. Ge. and T.S. Chung. .'Optimal Active Pawrr Flaw lncoroparating Power Flaw Control Needs in Felixbls AC 'Transmission Systems." /EE€ Tram. PmverSysreni . Vol. 14. No. 2. May 1999. pp. 738-744.

[8] S.N. Singh. A.K. David. "A New Approach for Placement of FACTS Devices in Open Power Markets." /E€€ Power Engimeerrrng Revmv. Septeniber200I. pp. 38-60,

[9] N. Li. Y. Xu. and H. Chen. --FACTS-Based Power Flaw Control in Interconnected Power Systems." IEEE Trans. Power Svsl.. vol. 15, no.1.257-262. Feb. 2000.

[10] Edris. A.S. Mehraban. M. Rahman. L. Gyugyi, S. Arabi. and T. Reitman. .Controlling the Flow of Kea1 and Reaoivr Power." IEEE Cornpurer Applrcafions m Power, 20-25. Jan 1998.

[11] S. Arabi and P. Kundur, "A versatilt facts device model for power flow and stability simulations." IEEE Trans. Power Sysr.. vol. I I , pp.19411950. Nov. 1996.

[12] X.-P. Zhang and E. Handschin. -0plimal power flow control by convener based facts controllers." in Proc. 7th Ini. Con/AC-DC PowerTrmsm.. London. U.K., Nov. 28-30,2001

[13] J. Mutale and G. Strbac, "Transmission Network Reinforcement Versus FACTS: An Econoniic Assessment." IEEE Tram. Power Sysr. vol. 15, no. 3.961-967. A U . 2nnn.

[14] E. Acha and H. Ambrir-Perez. -'FACTS devices modeling in optimal power tlow using Newton's method." in Pmc. 13fh Power Sysr Compirf. Con/, Trondheim. Norway, 1277-1284. June 28-July 2, 1999.
[15] E. Handschin and C. Lehmkoestrr. -'Optimal power tlow for deregulated systems with facts-devices." in Proc. 13rh Power Syst. Cotnput. Con/''rrondheim. Norway, 1270-1276, .June 28-July 2, 1999.

[16] J. B. Ward, "Equivalent circuits for power flow studies," AIEE Trans., vol. 68 , pp. 373-382, 1949.

[17] A. Monticelli, S. Deckmann, A. Garcia, and B. Stott, "Real-time external equivalents for static security analysis," IEEE Trans. Power App. Syst.,vol. PAS-98, pp. 498-508, Mar./Apr. 1979.

[18] Computer Techniques in power system analysis----M.A.Pai,p226,227

Sanjoy kumar Saha received the B.E and M.Tech degrees both in electrical engineering in 2003 and 2005 from Burdwan University, West Bengal, India and N.I.T, Durgapur, India, respectively. He is Sr.Lecturer in department of electrical engineering, Dr.B.C.Roy Engg. College, Durgapur (WB), India. His research interests are distributed generation planning and cost optimization. 